\title{
Willingness-to-pay and time-trade-off as the measures of burden of psoriasis: A pooled analysis of community, hospital, and web-based samples
}

\section{Yi Xiao}

Xiangya Hospital Central South University

\section{Meng Li}

University of Southern California

\section{Xin Guan}

Peking University Third Hospital

\section{Ping Sheng}

Dongfeng General Hospital

\section{Juan Tao}

Huazhong University of Science and Technology

\section{Meian He}

Huazhong University of Science and Technology

\section{Wu Zhu}

Xiangya Hospital Central South University

\section{Xiang Chen}

Xiangya Hospital Central South University

Minxue Shen ( $\nabla$ shenmx1988@csu.edu.cn)

Central South University Xiangya Hospital https://orcid.org/0000-0003-0441-9303

\section{Research}

Keywords: psoriasis, disease burden, willingness-to-pay, time-trade-off, health state utility

Posted Date: January 15th, 2020

DOl: https://doi.org/10.21203/rs.2.20894/v1

License: (c) (i) This work is licensed under a Creative Commons Attribution 4.0 International License. Read Full License 


\section{Abstract}

\section{Background}

The study aimed to compare willingness-to-pay (WTP) and health utility measured by time-trade-off (TTO) in psoriasis.

\section{Methods}

A pooled sample from a community-based cohort, a hospital, and an online registry were used. WTP was elicited for control and for cure. Psoriasis Area and Severity Index (PASI) and Dermatology Life Quality Index (DLQI) were used as references. Data distribution, concurrent validity, and area under the receiver operating characteristic curve (AUC) were compared.

Results

A total of 479 patients were enrolled. WTP for control demonstrated a positive association with PASI among mild psoriasis, while TTO showed an inverse correlation to DLQI. The AUC of WTP for control (0.66) was higher than that of TTO (0.48) in discriminating PASI>10, and the AUC of TTO (0.64) was higher than that of WTP for control (0.62) in discriminating DLQI>10.

Conclusion

WTP for control shows superiority in detecting objective severity, while TTO may be more sensitive in measuring impaired quality of life.

\section{Background}

Psoriasis, an immune-mediated chronic, relapsing, inflammatory, polygene genetic disease, is one of the most common skin disorders that affects $1-3 \%$ of the general population ${ }^{1}$. Although lots of therapies are available on the market, there is no cure for psoriasis so far $^{2}$. Patients with psoriasis not only suffer from itchiness and pain, but also experience considerable physical discomfort, social stigmata, and isolation, which adversely affects their quality of life (QoL) ${ }^{3}$. To date, several tools have been developed for measuring the QoL of psoriasis patients, and they can be divided into two main categories: intervalbased metrics and preference-based metrics ${ }^{4}$. The interval-based instruments, divided into generic (e.g., EQ-5D, SF-36), specialty-specific (e.g., the Dermatology Life Quality Index, DLQI), were well-established and widely used in dermatology. However, the application of preference-based metrics in dermatology was scarce.

Utility evaluates the desirability of certain health states. A commonly accepted assessment of utility is the time-trade-off (TTO) method ${ }^{5,6}$. The TTO method asks a person to make a trade-off between a shorter life expectancy without the condition and a longer life expectancy with the condition ${ }^{6}$. It captures the patient's preference over different health states, but is simpler than Standard Gamble (SG), which is 
based on the expected utility theory. In previous studies, the reported utility in skin diseases ranged from 0.64 in bullous disorders, to 0.907 in psoriasis, and 1.0 in alopecia, cosmetic concerns, and urticaria ${ }^{7}$. According to previous data, TTO seems to fail to distinguish the burden of different minor skin diseases 7 .

Willingness-to-pay (WTP), asking how much the responder is willing to pay for a hypothetical treatment, is also considered as a preference-based measure of disease burden in monetary terms ${ }^{7}$. The WTP method shows its potential in dermatology since the burden of many skin conditions tends to be transient and relatively mild and respondents are not willing to accept a shorter life expectancy without the skin disease in TTO or even a small probability of death in SG ${ }^{8}$. In the last two decades, the WTP metrics have been introduced in the field of dermatology and occasionally used in several skin diseases, such as psoriasis, atopic eczema, vitiligo, port wine stains, and rosacea ${ }^{9-16}$. Among which, both Schiffner R. et al. and Lundberg L. et al. reported TTO and WTP in psoriasis patients ${ }^{9,13}$; however, their properties of detecting different emphasis on the burden of psoriasis regarding data distribution, concurrent validity, and discrimination have not been thoroughly compared previously. Furthermore, to our knowledge, there is no previous study in dermatology that used community-based patients to capture the patients' WTP, and there is no literature that elicited WTP of patients with psoriasis in China before. Our study aims to estimate WTP and TTO of in a pooled sample of psoriasis patients, and to compare WTP and TTO regarding the sensitivity in measuring the burden of psoriasis.

\section{Methods}

\section{Study Design and Patients}

This was a cross-sectional study with a pooled sample of patients with diagnosed psoriasis recruited from one of the following settings (Figure S1).

1) The Dongfeng-Tongji cohort ${ }^{17}$ : The cohort recruited retired workers initially, and it began to recruit workers in-service since 2017 in addition to the previously recruited subjects. We used a sample of participants enrolled between July 2017 and October 2018. Certified dermatologists participated in the field survey and diagnosed skin diseases including psoriasis. Patients with psoriasis were included in the pooled analysis.

2) The dermatology clinic of a tertiary hospital in Changsha: Patients with psoriasis who gave their consent were consecutively recruited throughout 2017.

3) The Chinese Psoriasis Online Voluntary Registry ${ }^{18}$. The registry is a non-profit and coordinated effort to collect information directly from patients who are at least 18 years of age or older and have been diagnosed with psoriasis. With over 140,000 registered participants, the registry helped patients with psoriasis in the field of health education and social support. Unfortunately, this registry was not mentioned in a review of on psoriasis registry worldwide owing to a lack of publications ${ }^{19}$. We randomly 
invited a group of 250 patients to participate in this survey in September 2017. A subsidy was offered if a patient finished the survey to facilitate the response rate.

\section{Measures of Clinical Severity and QoL}

In this study, PASI was used as the gold standard to measure the clinical severity of psoriasis. Despite certain limitations such as the lack of consideration of symptoms, PASI is the most extensively used psoriasis clinical severity score and the most thoroughly validated instruments ${ }^{20}$. The following cut-off points were used: mild (0-10), moderate (11-15), and severe (>15). For the web-based patients, we used the self-reported clinical severity (0-10). The self-reported clinical severity was elicited by asking "how severe do you rate your clinical symptoms today (0-10)"; among which, "0" represents free from any psoriasis symptom, and " 10 " represents the most severe symptoms. The self-reported clinical severity was also categorized into three groups: mild (0-3), moderate (4-7), and severe (8-10).

In this study, DLQI ${ }^{21}$ was used to measure the QoL for all participants. DLQI is the most widely used QoL instruments in dermatology, and its psychometric properties in psoriasis have been widely validated. Unlike PASI score, DLQI may provide more subjective information and quality-of-life consideration beyond that described by PASI score. DLQI was categorised into three groups: mild (0-10), moderate (11-20), and severe (21-30).

\section{Measurement of WTP}

WTP was elicited using the contingent valuation method ${ }^{22}$, a widely used approach for the valuation of goods and services by potential consumers. Before the WTP amounts were elicited, the participants received education about psoriasis and that all payments were out-of-pocket with no insurance coverage. WTP was elicited for two scenarios: WTP for controlling the disease and WTP for curing the disease. For WTP $_{\text {cure, }}$ an open-ended question was used to ask about the total amount of money that they would be willing to pay to cure psoriasis. In order to directly compare WTP and TTO in percentage manner, we used relative WTP in this study. Rather than asking absolute money as WTP cure, $_{\text {, }}$ the WTP $_{\text {control }}$ was elicited by asking about the percentage of monthly income that the participant patients would like to pay to fully control the clinical symptoms of psoriasis. All hypothetical questions assumed no occurrence of side

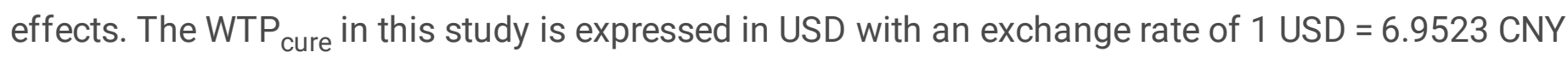
(2017).

\section{Measurement of Utility by TTO}

In this study, the TTO approach asks the subject to choose between a fixed guaranteed life duration in his or her current health with psoriasis or a shorter duration with no psoriasis. In order to elicit accurately, simply, and understandably, we obtained the life expectancy for Chinese population ${ }^{23}$ and informed participants their average life expectancies. Before elicitation, we thoroughly inquired the participants about their health status, including skin health and health status in general ${ }^{24}$. Participants were asked to choose from two options, option (A), remaining life-years (A years) with psoriasis in current status, or option (B), a shorter life duration (B years) without psoriasis or comorbid conditions. Ping-pong fashion 
was followed until arrival the maximum of time that patients are willing to trade-off for no-psoriasis status. The life duration in option $B$ is varied until the respondent is indifferent between option $A$ and option B. The TTO-derived utility of psoriasis is then calculated as $B /(A+B)$.

\section{Other Variables}

Age, gender, educational level, monthly income level, and duration of psoriasis were collected in the survey. Duration of psoriasis was dichotomized at the median (10 years) for the convenience of comparison.

\section{Statistical Analysis}

Medians and interquartile ranges (IQR) were used to describe the distribution of WTP and TTO, and Kruskal-Wallis $\mathrm{H}$ test was used to test the differences among groups. Concurrent validity was examined by correlations to PASI and DLQI. Spearman correlation coefficients were first estimated between WTP and TTO with PASI, DLQI, and self-reported severity. The locally estimated scatterplot smoothing (LOESS) method was then used to examine the nonlinear correlations ${ }^{25}$. The receiver operating characteristic (ROC) curve and AUC were used to compare the capability of WTP and TTO to discriminate patients with moderate-to-severe psoriasis from mild cases. A bootstrap method was used for the multiple comparisons of the AUCs among the groups. Income and duration of disease were adjusted for in models. Statistical analysis was performed using the R software; the R packages "ggplot2" and "pROC" were used for plotting ${ }^{26,27}$. A P $<0.05$ was considered statistically significant.

\section{Results}

\section{Patient Characteristics}

A total of 479 patients were enrolled from the Dongfeng-Tongji cohort (88 psoriasis patients out of 16,758 cohort participants; prevalence rate: 5.25/1000), the hospital (175), and the online registry (216 out of 250 distributed questionnaires; response rate: $86.4 \%$ ), respectively. The mean age of the participants was $44.2 \pm 13.3$ years. Patients from the Dongfeng-Tongji cohort were the oldest, had the longest duration of psoriasis, and had the highest QoL (Table 1). The mean PASI was $8.9 \pm 8.4$ and $4.2 \pm$ 6.0 for hospital and cohort patients, respectively. 
Table 1

Characteristics of the participants

\begin{tabular}{|c|c|c|c|c|}
\hline & \multicolumn{3}{|c|}{ Setting } & \multirow[b]{2}{*}{ Web } \\
\hline & Total & Community & Hospital & \\
\hline $\mathrm{N}$ & 479 & 88 & 175 & 216 \\
\hline Age, y & $44.2 \pm 13.3$ & $57.5 \pm 13.7$ & $38.3 \pm 13.0$ & $43.6 \pm 9.4$ \\
\hline \multicolumn{5}{|l|}{ Gender } \\
\hline Male & $318(66.4)$ & $56(63.6)$ & $65(50.4)$ & $151(57.6)$ \\
\hline Female & $161(33.6)$ & $32(36.4)$ & $64(49.6)$ & $111(42.4)$ \\
\hline \multicolumn{5}{|l|}{ Marital status } \\
\hline Unmarried & $114(23.8)$ & $8(9.1)$ & $26(14.8)$ & $80(37.0)$ \\
\hline Married & $345(72.0)$ & 77 (87.5) & $142(81.1)$ & $126(58.3)$ \\
\hline Divorced & $20(4.2)$ & $3(3.4)$ & $7(4.0)$ & $10(4.6)$ \\
\hline \multicolumn{5}{|l|}{ Educational level } \\
\hline Primary school and below & $40(8.3)$ & $11(12.5)$ & $21(12.0)$ & $8(3.7)$ \\
\hline Middle school & $207(43.2)$ & $21(23.9)$ & $79(45.1)$ & $107(49.5)$ \\
\hline High school & $157(32.8)$ & $24(27.3)$ & $61(34.9)$ & $72(33.3)$ \\
\hline College and above & $75(15.7)$ & $32(36.3)$ & $14(8.0)$ & $29(13.4)$ \\
\hline \multicolumn{5}{|l|}{ Monthly income, USD } \\
\hline$<216$ & $158(33.0)$ & $0(0.0)$ & $100(57.1)$ & $58(26.9)$ \\
\hline $216-431$ & 55 (11.5) & $6(6.8)$ & 38 (21.7) & $11(5.1)$ \\
\hline $432-863$ & $108(21.9)$ & $23(26.1)$ & $25(14.3)$ & $60(27.8)$ \\
\hline$\geq 864$ & $158(33.0)$ & $59(67.0)$ & $12(6.8)$ & $87(40.3)$ \\
\hline Course of psoriasis, y & $11.4 \pm 10.5$ & $18.6 \pm 15.3$ & $7.0 \pm 8.3$ & $11.3 \pm 7.9$ \\
\hline PASI (0-72) & $7.4 \pm 8.0$ & $4.2 \pm 6.0$ & $8.9 \pm 8.4$ & $\mathrm{~N} / \mathrm{A}$ \\
\hline Self-reported severity $(0-10)$ & $5.7 \pm 2.3$ & N/A & N/A & $5.7 \pm 2.3$ \\
\hline DLQI $(0-30)$ & $11.0 \pm 7.7$ & $5.2 \pm 6.1$ & $9.6 \pm 5.8$ & $14.0 \pm 7.7$ \\
\hline
\end{tabular}




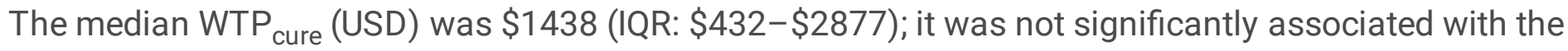
source of patient, income, and duration of psoriasis (Table 2). The median WTP control (\%) was $29 \%$ (IQR: $10-50 \%)$ of monthly income; it was slightly higher in women. The median of WTP control $_{\text {was lower in }}$ cohort-based patients (10\%; IQR: 0-30\%) than hospital-based patient (30\%; IQR: 10-50\%) and web-based patients (30\%; IQR: $20-50 \%)$, although the difference was not statistically significant $(P=0.115)$. 
Table 2

Medians and interquartile ranges of WTP and TTO across patient characteristics

\begin{tabular}{|c|c|c|c|c|c|c|}
\hline & \multicolumn{2}{|l|}{ WTP $_{\text {cure }}$, USD } & \multicolumn{2}{|c|}{$\mathrm{WTP}_{\text {control, }} \%$} & \multicolumn{2}{|l|}{ TTO } \\
\hline & Median (IQR) & $P$ & $\begin{array}{l}\text { Median } \\
\text { (IQR) }\end{array}$ & $P$ & $\begin{array}{l}\text { Median } \\
\text { (IQR) }\end{array}$ & $P$ \\
\hline \multicolumn{7}{|l|}{ Setting } \\
\hline Community & $\begin{array}{l}1438(0- \\
7192)\end{array}$ & 0.558 & $10(0-30)$ & 0.115 & $\begin{array}{l}0.90 \\
(0.85- \\
0.92)\end{array}$ & $<0.001$ \\
\hline Hospital & $\begin{array}{l}1438(432- \\
2877)\end{array}$ & & $\begin{array}{l}30(10- \\
50)\end{array}$ & & $\begin{array}{l}0.90 \\
(0.80- \\
0.92)\end{array}$ & \\
\hline Web & $\begin{array}{l}1438(432- \\
2877)\end{array}$ & & $\begin{array}{l}30(20- \\
50)\end{array}$ & & $\begin{array}{l}0.80 \\
(0.58- \\
0.90)\end{array}$ & \\
\hline \multicolumn{7}{|l|}{ Gender } \\
\hline Male & $\begin{array}{l}1438(432- \\
2877)\end{array}$ & 0.984 & $\begin{array}{l}30(12- \\
50)\end{array}$ & 0.005 & $\begin{array}{l}0.90 \\
(0.73- \\
0.93)\end{array}$ & 0.097 \\
\hline Female & $\begin{array}{l}1438(467- \\
2697)\end{array}$ & & $\begin{array}{l}32(20- \\
51)\end{array}$ & & $\begin{array}{l}0.87 \\
(0.70- \\
0.90)\end{array}$ & \\
\hline \multicolumn{7}{|l|}{ Marital status } \\
\hline Unmarried & $\begin{array}{l}1438(720- \\
2877)\end{array}$ & 0.195 & $\begin{array}{l}30(20- \\
51)\end{array}$ & 0.167 & $\begin{array}{l}0.85 \\
(0.65- \\
0.90)\end{array}$ & 0.219 \\
\hline Married & $\begin{array}{l}1150(432- \\
2877)\end{array}$ & & $\begin{array}{l}30(10- \\
50)\end{array}$ & & $\begin{array}{l}0.90 \\
(0.78- \\
0.92)\end{array}$ & \\
\hline Divorced & $\begin{array}{l}719(432- \\
1438)\end{array}$ & & $\begin{array}{l}40(19- \\
70)\end{array}$ & & $\begin{array}{l}0.90 \\
(0.48- \\
0.95)\end{array}$ & \\
\hline \multicolumn{7}{|l|}{ Educational level } \\
\hline $\begin{array}{l}\text { Primary school and } \\
\text { below }\end{array}$ & $\begin{array}{l}1438(647- \\
4315)\end{array}$ & 0.005 & $\begin{array}{l}30(10- \\
73)\end{array}$ & 0.886 & $\begin{array}{l}0.90 \\
(0.71- \\
0.90)\end{array}$ & 0.811 \\
\hline Middle school & $\begin{array}{l}719(432- \\
1438)\end{array}$ & & $\begin{array}{l}30(19- \\
50)\end{array}$ & & $\begin{array}{l}0.87 \\
(0.70- \\
0.90)\end{array}$ & \\
\hline
\end{tabular}




\begin{tabular}{|c|c|c|c|c|c|c|}
\hline & WTP $_{\text {cure, }}$ USD & & $\mathrm{WTP}_{\text {control, }}$ & & TTO & \\
\hline High school & $\begin{array}{l}1438(719- \\
2877)\end{array}$ & & $\begin{array}{l}30(20- \\
50)\end{array}$ & & $\begin{array}{l}0.90 \\
(0.70- \\
0.90)\end{array}$ & \\
\hline College and above & $\begin{array}{l}1438(503- \\
2877)\end{array}$ & & $\begin{array}{l}22(12- \\
51)\end{array}$ & & $\begin{array}{l}0.90 \\
(0.74- \\
0.95)\end{array}$ & \\
\hline \multicolumn{7}{|l|}{$\begin{array}{l}\text { Monthly income, } \\
\text { USD }\end{array}$} \\
\hline$<216$ & $\begin{array}{l}719(432- \\
2877)\end{array}$ & 0.232 & $\begin{array}{l}30(10- \\
50)\end{array}$ & 0.264 & $\begin{array}{l}0.90 \\
(0.73- \\
0.92)\end{array}$ & 0.275 \\
\hline $216-431$ & $\begin{array}{l}1438(575- \\
2877)\end{array}$ & & $\begin{array}{l}30(20- \\
56)\end{array}$ & & $\begin{array}{l}0.90 \\
(0.80- \\
0.90)\end{array}$ & \\
\hline $432-863$ & $\begin{array}{l}1438(575- \\
2877)\end{array}$ & & $\begin{array}{l}30(19- \\
50)\end{array}$ & & $\begin{array}{l}0.85 \\
(0.63- \\
0.90)\end{array}$ & \\
\hline$\geq 864$ & $\begin{array}{l}1438(719- \\
2877)\end{array}$ & & $\begin{array}{l}27(15- \\
50)\end{array}$ & & $\begin{array}{l}0.86 \\
(0.72- \\
0.95)\end{array}$ & \\
\hline \multicolumn{7}{|l|}{ Course of psoriasis } \\
\hline$<10 y$ & $\begin{array}{l}1438(432- \\
2877)\end{array}$ & 0.782 & $\begin{array}{l}30(10- \\
50)\end{array}$ & 0.370 & $\begin{array}{l}0.90 \\
(0.70- \\
0.92)\end{array}$ & 0.218 \\
\hline$\geq 10 y$ & $\begin{array}{l}1438(432- \\
2877)\end{array}$ & & $\begin{array}{l}25(10- \\
50)\end{array}$ & & $\begin{array}{l}0.85 \\
(0.70- \\
0.90)\end{array}$ & \\
\hline
\end{tabular}

The median TTO was 0.9 (IQR: 0.70-0.92). Community patients (0.90; IQR: 0.85-0.92) and hospital patients (0.90; IQR: 0.80-0.92) showed significantly higher TTO than web patients (0.80; IQR: 0.58-0.90). TTO was not associated with income and duration of psoriasis.

\section{Data Distribution}

The WTP $_{\text {cure }}$ showed a normal distribution after logarithmic transformation, while TTO showed a right-

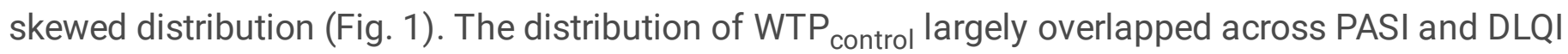
groups. The Kruskal-Wallis tests showed consistent results with the visual description: the medians of WTP $_{\text {cure }}$ were not significantly different across PASI or DLQI groups (Table S1). In contrast, the

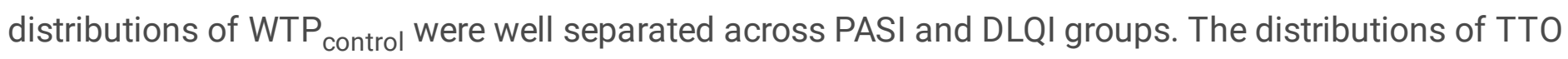
overlapped across PASI groups, but were well separated across DLQI groups. Statistical tests also showed consistent results (Table S1). The medians of WTP and TTO were not significantly different 


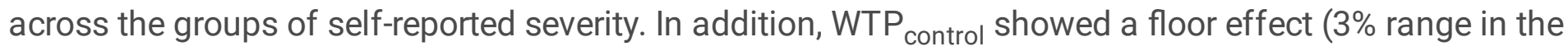
lower $10 \%$ centile) while TTO showed a ceiling effect ( 0.05 range in the upper $10 \%$ centile, Table S2).

\section{Concurrent validity}

WTP $_{\text {control }}$ was significantly associated with PASI $(r=0.36, P<0.001)$ but not with DLQI, while TTO was significantly associated with DLQI $(r=-0.20, P=0.001)$, but not with PASI (Table S3). Partial correlation coefficients adjusting for income and duration of psoriasis were consistent with the unadjusted results.

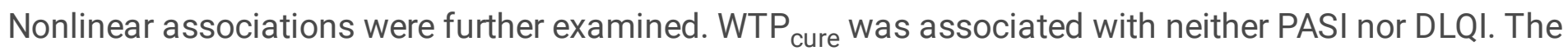

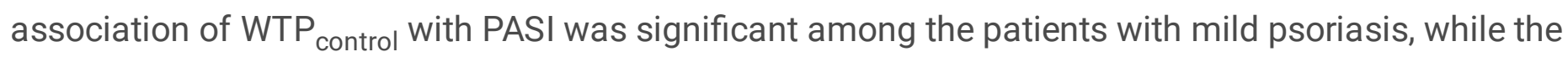
confidence interval of the curve became very wide among those with moderate-to-severe psoriasis (Fig. 2). TTO was significantly inversely associated with DLQI, but was not correlated with PASI.

\section{Discrimination}

The AUC of WTP control $(A U C=0.66,95 \% \mathrm{Cl}: 0.58-0.74)$ was statistically significant in discriminating PASI $>10$, while the AUCs of WTP cure $(A U C=0.52,95 \% \mathrm{Cl}: 0.44-0.61)$ and TTO (AUC $=0.48,95 \%$ Cl: $0.40-0.60)$ were not significant (Table 3 \& Figure S2). In contrast, the AUC of TTO (AUC $=0.64,95 \% \mathrm{Cl}: 0.58-0.70$ ) was significantly larger than that of WTP $_{\text {cure }}(A U C=0.55,95 \% \mathrm{Cl}: 0.58-0.74)$ and WTP $_{\text {control }}(A U C=0.62$, $95 \% \mathrm{Cl}: 0.56-0.67)$ in the discrimination for DLQI > 10, using the bootstrap method.

Table 3

Comparisons of the area under the receiver operating characteristic curves using the bootstrap method

\begin{tabular}{|c|c|c|c|c|c|c|}
\hline & \multicolumn{3}{|c|}{ PASI $>10$} & \multicolumn{3}{|c|}{ DLQI > 10} \\
\hline & AUC & $95 \% \mathrm{Cl}$ & $P$ & AUC & $95 \% \mathrm{Cl}$ & $P$ \\
\hline \multicolumn{7}{|l|}{ AUCs } \\
\hline WTP cure, USD & 0.52 & $0.44-0.61$ & 0.457 & 0.54 & $0.48-0.60$ & 0.852 \\
\hline WTP $_{\text {control, }} \%$ & 0.67 & $0.58-0.74$ & $<0.001$ & 0.62 & $0.56-0.67$ & $<0.001$ \\
\hline TTO & 0.48 & $0.40-0.60$ & 0.768 & 0.64 & $0.58-0.70$ & $<0.001$ \\
\hline \multicolumn{7}{|l|}{ Comparison of AUCs } \\
\hline WTP $_{\text {cure }}$ vs. WTP control & & & 0.047 & & & 0.017 \\
\hline WTP $_{\text {cure }}$ vs. TTO & & & 0.272 & & & $<0.001$ \\
\hline WTP $_{\text {control }}$ vs. TTO & & & 0.008 & & & 0.031 \\
\hline
\end{tabular}




\section{Discussion}

For the first time, the study reported WTP of Chinese psoriasis patients and, investigated WTP and utilities (TTO) in real-world patients with psoriasis. To our knowledge, the previous literature on WTP and TTO of psoriasis patients were based on hospital sample; and this may lead to a bias to capture the overview of impaired QoL in patients with psoriasis. This study included both community and hospital samples, providing a more comprehensive estimate of the burden of psoriasis in China. The median TTO estimate of our study population was 0.9 (IQR: 0.70-0.92), which was close to the upper limit of previous estimates ${ }^{7}$. Since our pooled sample is more representative for the patient population in China, the utilities elicited from the presenting study are more convincing and valuable.

This study was among the first to systematically compare the properties of WTP and TTO as measures for the burden of psoriasis regarding data distribution, ceiling and floor effects, linear and nonlinear correlation, and capability of discrimination. In order to compare WTP and TTO in a more unified and direct approach, we used relative WTP in the present study since absolute WTP fails to generalised outside China. The results indicated that the WTP for monthly control (\%) was highly correlated with the clinical severity of psoriasis (PASI) while TTO was highly correlated with non-preference-based QoL measure (DLQI). WTP cure in absolute monetary terms was relevant to neither the clinical severity nor the QoL, which was in accordance with the conclusions from previous studies that relative WTP might be a better indicator compared with absolute WTP ${ }^{7}$.

In our study, TTO showed a unique capability of representing QoL across all groups. TTO, a classic method to elicit health state utility, has been widely used across diseases. By asking people "how many years of current life with a certain illness they would like to give up in exchange for the rest living free from this condition," it allows direct comparisons of QoL across different disease areas. In this study, TTO demonstrated a consistent positive correlation with DLQI in patients with varying levels of scores. This result is interesting but not surprising. Several previous studies substantiated that DLQI is good at detecting impaired QoL ${ }^{28,29}$. The more the patients felt embarrassed or self-conscious, or ashamed of their physical appearance, or experienced difficulties in social interactions, the more they are willing to accept a shorter life expectancy without psoriasis. However, in our study, TTO showed ceiling effect and failed to show superiority in detecting clinical severity compared to WTP $\mathrm{W}_{\text {control, }}$, especially when psoriasis is in a mild status. Since TTO serves as a ruler of comparison across all diseases, the tool cannot be fine enough to detect nuances, especially in skin diseases with rare death or disability. We also identified a ceiling effect that the range of TTO in the upper $10 \%$ of patients with psoriasis was only 0.05 . Indeed, even when their QoL was truly impaired, it was hard for patients with a skin disease to trade their life in return of free from cutaneous signs and symptoms.

Consistent with the suggestions from Chen S. et al. ${ }^{8}$, we continue to advocate the application of the WTP metrics in psoriasis. Indeed, WTP, as a monetary indicator, has inherent flaws such as its correlation to

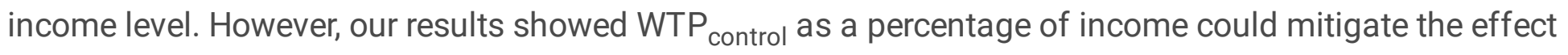
of income originally existing in absolute WTP. In our study, the distribution of WTP for disease control (\%) 
was well separated across PASI and DLQI groups, especially among those in a mild disease status(PASI< 10), indicating that WTP rather than TTO estimates can better reflect disease burden in mild psoriasis. Paralleled with the clinical severity of psoriasis, the dynamic conceptualization of the money paid out-ofpocket for a possible treatment may be more straightforward and sensitive to the patients when comparing with TTO. These findings, if generalised, could provide further evidence to support the use of relative WTP to measure the burden of disease in dermatology, since most of the skin diseases are relatively mild and temporary compared to other major diseases.

There are limitations in this study. First, there is a lack of gold standard for measuring the burden of psoriasis. However, PASI and DLQI itself are generally acknowledged tools to capture the burden of patients with psoriasis, and they are complementary to each other in the evaluation of psoriasis ${ }^{30}$. As a result, PASI and DLQI were used as the references. Second, the sample size of patients with PASI > 10 was relatively small, and a robust conclusion could hardly be drawn among those with moderate-to-severe psoriasis. Further study is needed to verify our findings in larger samples and different populations. Despite the limitations, our study can serve as an essential contribution to the existing literature. By the use of a pooled sample from a population-based cohort, a tertiary hospital, and the biggest psoriasis online registry in China, the current study reports the first data of WTP of psoriasis patients in China and can be more comprehensive than hospital-based studies. The characteristics of the patients varied substantially across the different sources of sample, which further demonstrates the heterogeneity of patients in different settings and underscores the importance of including different patient populations in the sample. More importantly, we found that WTP $_{\text {control }}$ is superior in detecting minor clinical severity status of psoriasis compared to TTO, while TTO is a more sensitive indicator for the impaired healthrelated QoL associated with psoriasis. In general, relative WTP and TTO are both recommended to depict the complete picture of the burden of psoriasis regarding multifaceted emphases.

\section{References}

1. Parisi R, Symmons DP, Griffiths CE, et al. Global epidemiology of psoriasis: a systematic review of incidence and prevalence. J Invest Dermatol. 2013;133(2):377-385.

2. Bhosle MJ, Kulkarni A, Feldman SR, Balkrishnan R. Quality of life in patients with psoriasis. Health Qual Life Outcomes. 2006;4:35.

3. Parrish L. Psoriasis: symptoms, treatments and its impact on quality of life. Br J Community Nurs. 2012;17(11):524, 526, 528.

4. Kini SP, DeLong LK. Overview of health status quality-of-life measures. Dermatol Clin. 2012;30(2):209-221, xiii.

5. Chen SC, Bayoumi AM, Soon SL, et al. A catalog of dermatology utilities: a measure of the burden of skin diseases. J Investig Dermatol Symp Proc. 2004;9(2):160-168.

6. Drummond MF, Sculpher MJ, Claxton K, Stoddart GL, Torrance GW. Methods for the economic evaluation of health care programmes (4th edition). Oxford University Press; 2015. 
7. Seidler AM, Kini SP, DeLong LK, Veledar E, Chen SC. Preference-based measures in dermatology: an overview of utilities and willingness to pay. Dermatol Clin. 2012;30(2):223-229, xiii.

8. Seidler AM, Bayoumi AM, Goldstein MK, Cruz PD, Jr., Chen SC. Willingness to pay in dermatology: assessment of the burden of skin diseases. J Invest Dermatol. 2012;132(7):1785-1790.

9. Lundberg L, Johannesson M, Silverdahl M, Hermansson C, Lindberg M. Quality of life, health-state utilities and willingness to pay in patients with psoriasis and atopic eczema. Br J Dermatol. 1999;141(6):1067-1075.

10. Radtke MA, Schafer I, Gajur A, Langenbruch A, Augustin M. Willingness-to-pay and quality of life in patients with vitiligo. Br J Dermatol. 2009;161(1):134-139.

11. Delfino M, Jr., Holt EW, Taylor CR, Wittenberg E, Qureshi AA. Willingness-to-pay stated preferences for 8 health-related quality-of-life domains in psoriasis: a pilot study. J Am Acad Dermatol. 2008;59(3):439-447.

12. Beikert FC, Langenbruch AK, Radtke MA, Kornek T, Purwins S, Augustin M. Willingness to pay and quality of life in patients with atopic dermatitis. Arch Dermatol Res. 2014;306(3):279-286.

13. Schiffner R, Brunnberg S, Hohenleutner U, Stolz W, Landthaler M. Willingness to pay and time tradeoff: useful utility indicators for the assessment of quality of life and patient satisfaction in patients with port wine stains. Br J Dermatol. 2002;146(3):440-447.

14. Beikert FC, Langenbruch AK, Radtke MA, Augustin M. Willingness to pay and quality of life in patients with rosacea. J Eur Acad Dermatol Venereol. 2013;27(6):734-738.

15. Schiffner R, Schiffner-Rohe J, Gerstenhauer M, Hofstadter F, Landthaler M, Stolz W. Willingness to pay and time trade-off: sensitive to changes of quality of life in psoriasis patients? Br J Dermatol. 2003;148(6):1153-1160.

16. Schmitt J, Meurer M, Klon M, Frick KD. Assessment of health state utilities of controlled and uncontrolled psoriasis and atopic eczema: a population-based study. $\mathrm{Br} \mathrm{J}$ Dermatol. 2008;158(2):351-359.

17. Wang F, Zhu J, Yao P, et al. Cohort Profile: the Dongfeng-Tongji cohort study of retired workers. Int J Epidemiol. 2013;42(3):731-740.

18. International Research Center of Medicinal Administration. A survey of burden of disease and quality of life in patients with psoriasis in China. 2018; https://img.dxycdn.com/biz/thyroid2017_files/edit/1018105030586.pdf. Accessed 25 Nov 2018.

19. Eissing L, Rustenbach SJ, Krensel M, et al. Psoriasis registries worldwide: systematic overview on registry publications. J Eur Acad Dermatol Venereol. 2016;30(7):1100-1106.

20. Puzenat E, Bronsard V, Prey $S$, et al. What are the best outcome measures for assessing plaque psoriasis severity? A systematic review of the literature. J Eur Acad Dermatol Venereol. 2010;24:1016.

21. Finlay AY, Khan GK. Dermatology Life Quality Index (DLQI)-a simple practical measure for routine clinical use. Clin Exp Dermatol. 1994;19(3):210-216. 
22. Mitchell RC, Carson RT. Using surveys to value public goods: the contingent valuation method. RFF Press; 2013.

23. National Bureau of Statistics of China. China Statistical Yearbook 2016. Beijing: China Statistics Press; 2017: http://www.stats.gov.cn/tjsj/ndsj/2016/indexch.htm.

24. Harris RA, Nease RF, Jr. The importance of patient preferences for comorbidities in cost-effectiveness analyses. J Health Econ. 1997;16(1):113-119.

25. Cleveland WS, Devlin SJ. Locally weighted regression: an approach to regression analysis by local fitting. J Am Stat Assoc. 1988;83:596-610.

26. Wickham H. ggplot2: Elegant Graphics for Data Analysis. Springer; 2009.

27. Robin X, Turck N, Hainard A, et al. pROC: an open-source package for R and S+ to analyze and compare ROC curves. BMC Bioinformatics. 2011;12:77.

28. Lewis V, Finlay AY. 10 years experience of the Dermatology Life Quality Index (DLQI). J Investig Dermatol Symp Proc. 2004;9(2):169-180.

29. Hongbo Y, Thomas CL, Harrison MA, Salek MS, Finlay AY. Translating the science of quality of life into practice: What do dermatology life quality index scores mean? J Invest Dermatol. 2005;125(4):659-664.

30. Kimball AB, Krueger G, Woolley JM. The Dermatology Life Quality Index (DLQI) provides qualitatively different information from the PASI. J Am Acad Dermato/ 2004;50(3):P156-P156.

\section{List Of Abbreviations}

AUC: area under the curve. DLQI: Dermatology Life Quality Index. IQR: interquartile range. LOESS: locally estimated scatterplot smoothing. PASI: Psoriasis Area and Severity Index. QoL: quality of life. ROC: receiver operating characteristic. TTO: time-trade-off. WTP: willingness-to-pay.

\section{Declarations}

\section{Ethics approval and consent to participate}

This study was conducted according to the guidelines laid down in the Declaration of Helsinki. All procedures involving patients were approved by the institutional research ethics boards of Xiangya Hospital, Central South University (Changsha, China). Written (cohort and hospital participants) or electronic (web participants) informed consent was obtained from all participants.

\section{Consent for publication}

No patient's individual data was presented.

\section{Availability of data and materials}

The data is avalaible upon appropriate request to the corresponding authors. 


\section{Competing interests}

The authors declare no competing interest.

\section{Funding}

This work was supported by the National Key Research \& Development Project of China "Precision Medicine Initiative" (grant\# 2016YFC0900802), and the Key Research \& Development Project of Hunan Province (grant\# 2018SK260).

\section{Authors' contribution}

All authors participated in field survey and data collection. Y.X., M.S., and X.C. designed the study. M.S. analyzed the data. Y.X. wrote the manuscript. M.L., X.G., P.S., J.T., M.H., W.Z., and X.C. interpreted the data and critically revised the manuscript. X.C. and M.S. obtained the funding.

\section{Acknowledgements}

The authors would thank the following investigators and dermatologists that participated in the study. Xiangya Hospital, Central South University: Liping Chen, Menglin Chen, Yihuan Gong, Junweichen Guo, Xiaoyan Huang, Yuzhou Huang, Danrong Jing, Jia Li, Dan Liu, Yuyan Ouyang, Xiangmei Ren, Ke Sha, Yajie Tang, Yan Tang, Ben Wang, Yaling Wang, Taolin Yu, Zhixiang Zhao, Xingyu Zhang, Bolan Zheng, Lei Zhou, and Qian Zhou. Union Hospital, Tongji Medical College, Huazhong University of Science and Technology: Xiuling Liu, Yingli Nie, Huinan Suo, Bingbing Wang, Zhen Ye, Jing Yuan, Li Zhu, and Yanming Zhu. Sinopharm Dongfeng General Hospital, Hubei University of Medicine: Yaqi Dong, Yuxia Hu, Zhenzhen Huang, Yang Jiang, Cuiting Li, Han Liu, Limin Luo, Jiao Wei, Yuran Wu, and Xiao Xiong.

\section{Supplementary Information}

Table S1. Medians and interquartile ranges of WTP and TTO among patients with different severities of psoriasis.

Table S2. Ceiling and floor effects of WTP and TTO in patients with psoriasis

Table S3. Spearman correlation coefficients between WTP and TTO and PASI and DLQI.

Figure S1. Flowchart of the study.

Figure S2. Receiver operating characteristic curves. The blue lines signify the ROC curves, and the light blue bands signify the $95 \%$ confidence intervals of the sensitivities. (A) ROC curve of WTP cure (USD) to discriminate PASI>10. (B) ROC curve of WTP control (\% monthly income) to discriminate PASI $>10$. (C) The ROC curve of TTO to discriminate PASI>10. (D) ROC curve of WTP $P_{\text {cure }}$ to discriminate DLQI>10. (E) ROC

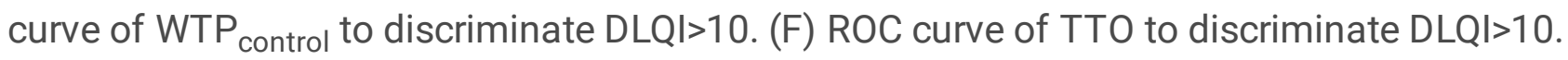




\section{Figures}
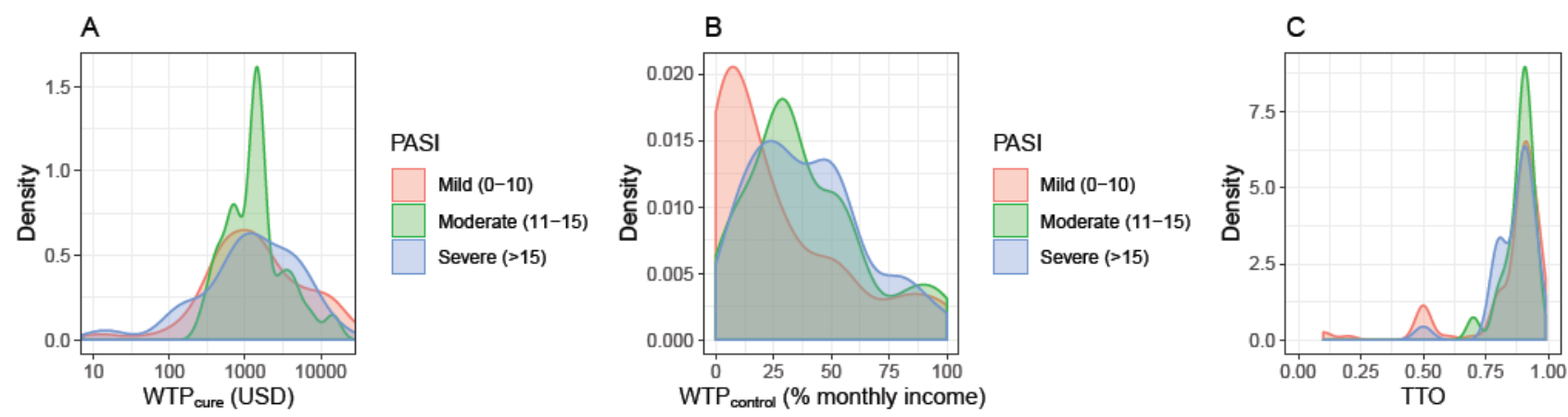

PASI

Mild (0-10)

Moderate (11-15)

Severe $(>15)$
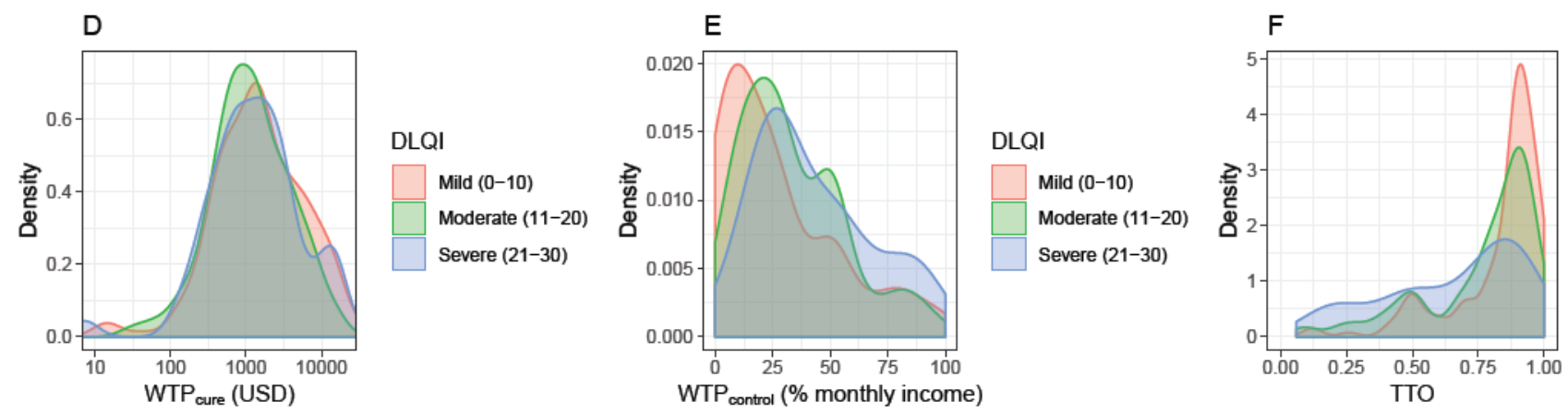

DLQI

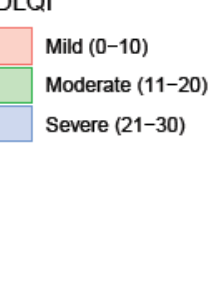

Mild (0-10) Moderate (11-20) Severe (21-30)

\section{Figure 1}

Distribution of WTP and TTO by the severity of psoriasis. (A) Distribution of WTPcure (USD), stratified by PASI group (mild, moderate, severe); logarithmic coordinate axis was used for WTPcure. (B) Distribution of WTPcontrol (\% monthly income), stratified by PASI group. (C) Distribution of TTO, stratified by PASI group. (D) Distribution of WTPcure, stratified by DLQI group (mild, moderate, severe). (E) Distribution of WTPcontrol, stratified by DLQI group. (F) Distribution of TTO, stratified by DLQI group. 

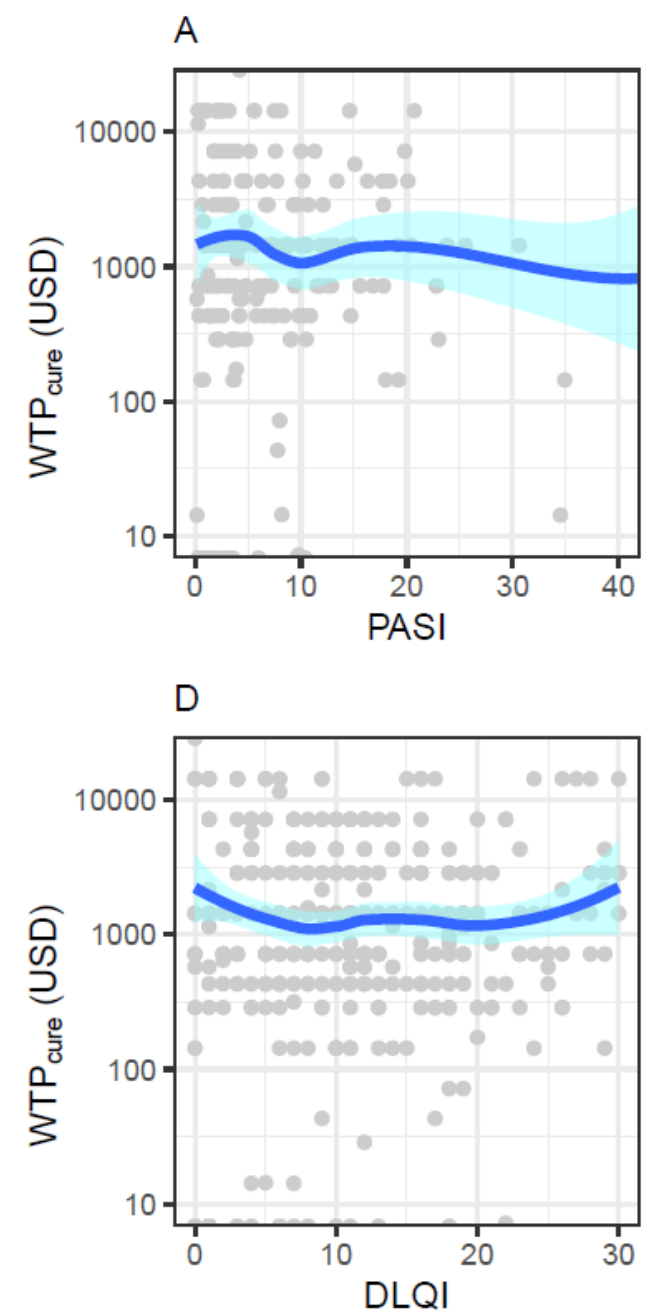
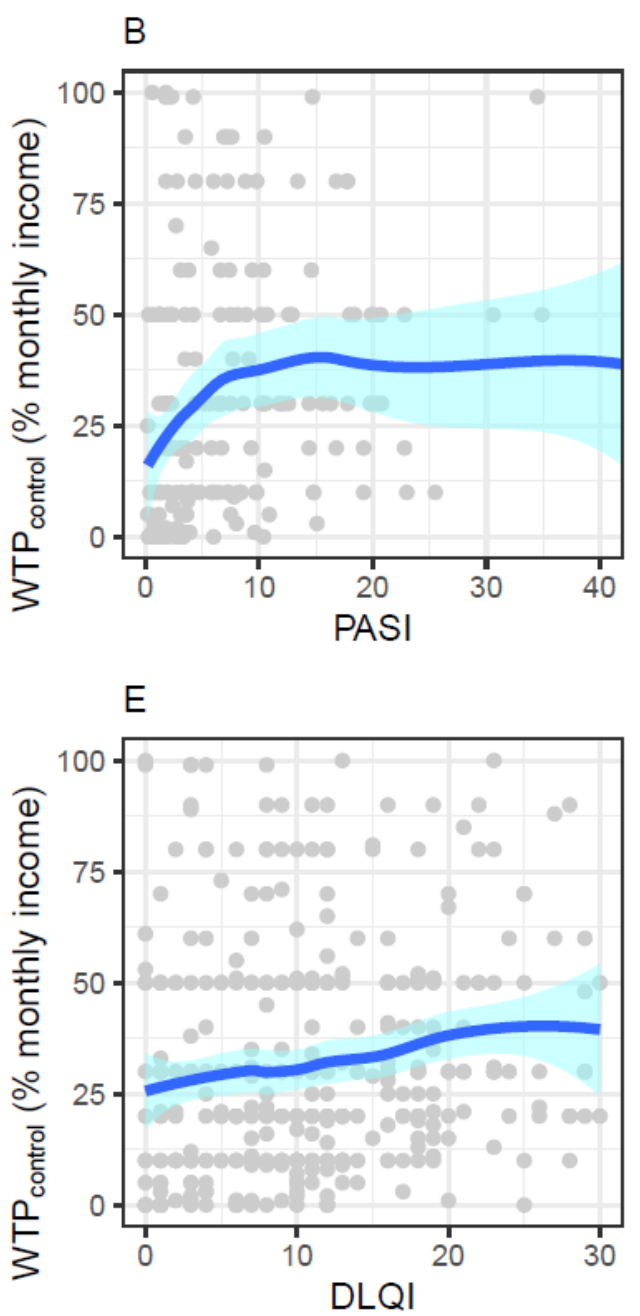
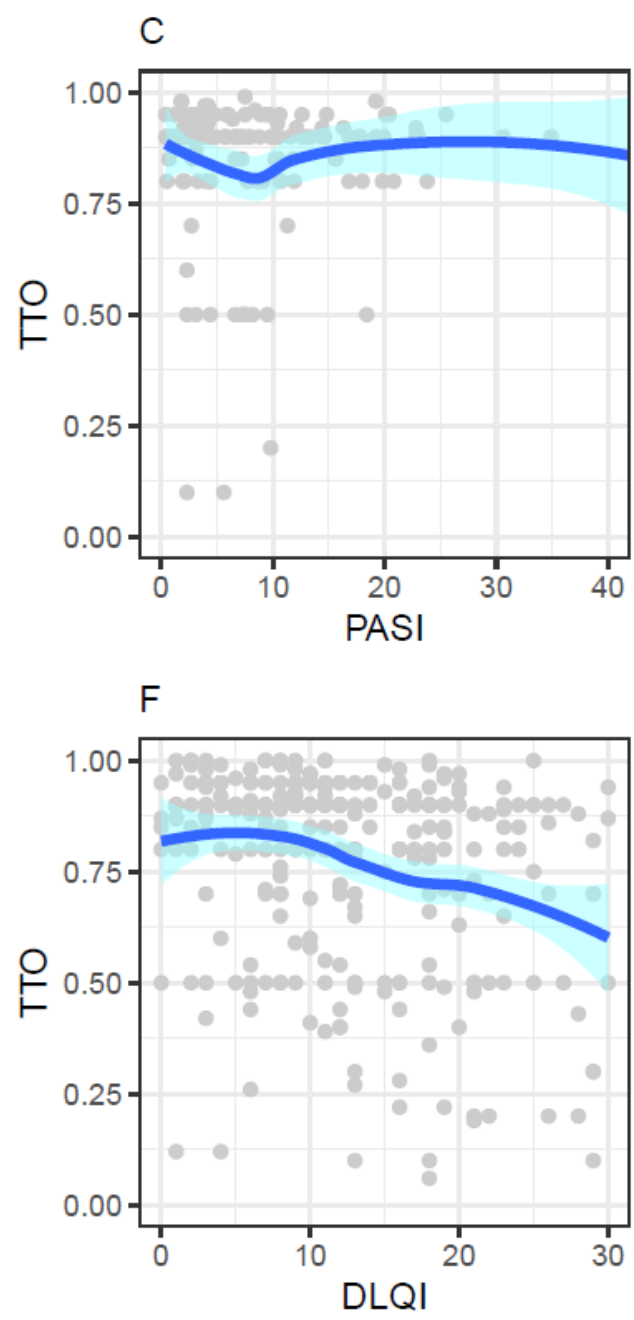

Figure 2

Correlation of WTP and TTP to PASI and DLQI using a locally estimated scatterplot smoothing method. The blue lines signify the estimated curves and the light blue bands signify the $95 \%$ confidence intervals. (A) Association of WTPcure (USD) with PASI; logarithmic coordinate axis was used for WTPcure. (B) Association of WTPcontrol (\% monthly income) with PASI. (C) Association of TTO with PASI. (D) Association of WTPcure with DLQI. (E) Association of WTPcontrol with DLQI. (F) Association of TTO with DLQI.

\section{Supplementary Files}

This is a list of supplementary files associated with this preprint. Click to download.

- Supplementaryfiles.pdf 